\title{
Quotient probabilistic normed spaces and completeness results
}

\author{
BERNARDO LAFUERZA-GUILLÉN ${ }^{1}$, DONAL O'REGAN ${ }^{2}$ and \\ REZA SAADATI ${ }^{3}$
}

${ }^{1}$ Departamento de Estadística y Matemática Aplicada, Universidad de Almería, 04120 Almería, Spain

${ }^{2}$ Department of Mathematics, National University of Ireland, Galway, Ireland

${ }^{3}$ Faculty of Sciences, University of Shomal, Amol, Iran

E-mail: blafuerz@ual.es; donal.oregan@nuigalway.ie; rsaadati@eml.cc

MS received 8 December 2005; revised 30 March 2006

Abstract. We introduce the concept of quotient in PN spaces and give some examples. We prove some theorems with regard to the completeness of a quotient.

Keywords. Probabilistic normed space; probabilistic norm; triangle functions; quotient probabilistic normed space; $\sigma$-product.

\section{Introduction}

In the literature devoted to the theory of probabilistic normed spaces (PN spaces, briefly), topological and completeness questions, boundedness and compactness concepts $[4,5$, 7], linear operators, probabilistic norms for linear operators [6], product spaces [3] and fixed point theorems have been studied by various authors. However quotient spaces of PN spaces have never been considered. This note is a first attempt to fill this gap.

The present paper is organized as follows. In $\S 2$ all necessary preliminaries are recalled and notation is established. In $\S 3$, the quotient space of a PN space with respect to one of its subspaces is introduced and its properties are studied. Finally, in $\S 4$, we investigate the completeness relationship among the PN spaces considered.

\section{Definitions and preliminaries}

In the sequel, the space of all probability distribution functions (briefly, d.f.'s) is $\Delta^{+}=$ $\{F: \mathbf{R} \cup\{-\infty,+\infty\} \longrightarrow[0,1]: F$ is left-continuous and non-decreasing on $\mathbf{R}, F(0)=0$ and $F(+\infty)=1\}$ and the subset $D^{+} \subseteq \Delta^{+}$is the set $D^{+}=\left\{F \in \Delta^{+}: l^{-} F(+\infty)=1\right\}$. Here $l^{-} f(x)$ denotes the left limit of the function $f$ at the point $x, l^{-} f(x)=\lim _{t \rightarrow x^{-}} f(t)$. The space $\Delta^{+}$is partially ordered by the usual point-wise ordering of functions, i.e., $F \leq G$ if and only if $F(x) \leq G(x)$ for all $x$ in $\mathbf{R}$. The maximal element for $\Delta^{+}$in this order is the d.f. given by

$$
\varepsilon_{0}= \begin{cases}0, & \text { if } x \leq 0 \\ 1, & \text { if } x>0\end{cases}
$$


Also the minimal element for $\Delta^{+}$in this order is the d.f. given by

$$
\varepsilon_{\infty}= \begin{cases}0, & \text { if } x \leq \infty, \\ 1, & \text { if } x=\infty .\end{cases}
$$

We assume that $\Delta$ is metrized by the Sibley metric $d_{S}$, which is the modified Lévy metric $[8,9]$. If $F$ and $G$ are d.f.'s and $h$ is in $(0,1]$, let $(F, G ; h)$ denote the condition

$$
F(x-h)-h \leq G(x) \leq F(x+h)+h
$$

for all $x$ in $(-1 / h, 1 / h)$. Then the modified Lévy metric (Sibley metric) is defined by

$$
d_{S}(F, G):=\inf \{h>0 \text { : both }(F, G ; h) \text { and }(G, F ; h) \text { hold }\} .
$$

For any $F$ in $\Delta^{+}$,

$$
\begin{aligned}
d_{S}\left(F, \varepsilon_{0}\right) & =\inf \left\{h>0:\left(F, \varepsilon_{0} ; h\right) \text { holds }\right\} \\
& =\inf \left\{h>0: F\left(h^{+}\right)>1-h\right\},
\end{aligned}
$$

and for any $t>0$,

$$
F(t)>1-t \Longleftrightarrow d_{S}\left(F, \varepsilon_{0}\right)<t .
$$

It follows that, for every $F, G$ in $\Delta^{+}$,

$$
F \leq G \Longrightarrow d_{S}\left(G, \varepsilon_{0}\right) \leq d_{S}\left(F, \varepsilon_{0}\right) .
$$

A sequence $\left(F_{n}\right)$ of d.f.'s converges weakly to a d.f. $F$ if and only if the sequence $\left(F_{n}(x)\right)$ converges to $F(x)$ at each continuity point $x$ of $F$. For the proof of the next theorem see Theorem 4.2 .5 of [8].

Theorem 2.1. Let $\left(F_{n}\right)$ be a sequence of functions in $\Delta$, and let $F$ be in $\Delta$. Then $F_{n} \rightarrow F$ weakly if and only if $d_{S}\left(F_{n}, F\right) \rightarrow 0$.

\section{DEFINITION 2.2}

A triangular norm $T$ (briefly, a t-norm) is an associative binary operation on $[0,1]$ (henceforth, $I)$ that is commutative, nondecreasing in each place, such that $T(a, 1)=a$ for all $a \in I$.

\section{DEFINITION 2.3}

Let $T$ be a binary operation on $I$. Denote by $T^{*}$ the function defined by $T^{*}(a, b):=$ $1-T(1-a, 1-b)$ for all $a, b \in I$. If $T$ is a $t$-norm, then $T^{*}$ will be called the $t$-conorm of $T$. A function $S$ is a $t$-conorm if there is a $t$-norm $T$ such that $S=T^{*}$.

Clearly, $T^{*}$ is itself a binary operation on $I$, and $T^{* *}=T$. Instances of such $t$-norms and $t$-conorms are $M$ and $M^{*}$, respectively, defined by $M(x, y)=\min (x, y)$ and $M^{*}(x, y)=$ $\max (x, y)$.

\section{DEFINITION 2.4}

A triangle function $\tau$ is an associative binary operation on $\Delta^{+}$that is commutative, nondecreasing in each place, and has $\varepsilon_{0}$ as identity. 
Also we let $\tau^{1}=\tau$ and

$$
\tau^{n}\left(F_{1}, \ldots, F_{n+1}\right)=\tau\left(\tau^{n-1}\left(F_{1}, \ldots, F_{n}\right), F_{n+1}\right) \text { for } n \geq 2 .
$$

Let $T$ be a left-continuous $t$-norm and $T^{*}$ a right-continuous $t$-conorm. Then instances of such triangle functions are $\tau_{T}$ and $\tau_{T^{*}}$ defined for all $F, G \in \Delta^{+}$and every $x \in \mathbf{R}^{+}$, respectively, by

$$
\tau_{T}(F, G)(x)=\sup \{T(F(u), G(v)) \mid u+v=x\}
$$

and

$$
\tau_{T^{*}}(F, G)(x)=\ell^{-} \inf \left\{T^{*}(F(u), G(v)) \mid u+v=x\right\} .
$$

The triangular function $\tau$ is said to be Archimedean on $\Delta^{+}$if $\tau(F, G)<F$ for any $F, G$ in $\Delta^{+}$, such that $F \neq \varepsilon_{\infty}$ and $G \neq \varepsilon_{0}$.

\section{DEFINITION 2.5}

Let $\tau_{1}, \tau_{2}$ be two triangle functions. Then $\tau_{1}$ dominates $\tau_{2}$, and we write $\tau_{1} \gg \tau_{2}$, if for all $F_{1}, F_{2}, G_{1}, G_{2} \in \Delta^{+}$,

$$
\tau_{1}\left(\tau_{2}\left(F_{1}, G_{1}\right), \tau_{2}\left(F_{2}, G_{2}\right)\right) \geq \tau_{2}\left(\tau_{1}\left(F_{1}, F_{2}\right), \tau_{1}\left(G_{1}, G_{2}\right)\right) .
$$

In 1993, Alsina, Schweizer and Sklar [1] gave a new definition of a probabilistic normed space as follows:

\section{DEFINITION 2.6}

A probabilistic normed space, briefly a PN space, is a quadruple $\left(V, v, \tau, \tau^{*}\right)$ in which $V$ is a linear space, $\tau$ and $\tau^{*}$ are continuous triangle functions with $\tau \leq \tau^{*}$ and $\nu$, the probabilistic norm, is a map $v: V \rightarrow \Delta^{+}$such that

(N1) $v_{p}=\varepsilon_{0}$ if and only if $p=\theta, \theta$ being the null vector in $V$;

(N2) $v_{-p}=v_{p}$ for every $p \in V$;

(N3) $v_{p+q} \geq \tau\left(v_{p}, v_{q}\right)$ for all $p, q \in V$;

(N4) $v_{p} \leq \tau^{*}\left(v_{\alpha p}, v_{(1-\alpha) p}\right)$ for every $\alpha \in[0,1]$ and for every $p \in V$.

If, instead of (N1), we only have $v_{\theta}=\varepsilon_{0}$, then we shall speak of a probabilistic pseudo normed space, briefly a PPN space. If the inequality (N4) is replaced by the equality $v_{p}=\tau_{M}\left(v_{\alpha p}, v_{(1-\alpha) p}\right)$, then the PN space is called a Šerstnev space; in this case, condition stronger than (N2) holds, namely

$$
v_{\lambda p}=v_{p}\left(\frac{j}{|\lambda|}\right), \quad \forall \lambda \neq 0, \forall p \in V
$$

here $j$ is the identity map on $\mathbf{R}$. A Šerstnev space is denoted by $(V, v, \tau)$.

There is a natural topology in a PN space $\left(V, v, \tau, \tau^{*}\right)$, called the strong topology; it is defined, for $t>0$, by the neighbourhoods

$$
N_{p}(t):=\left\{q \in V: d_{S}\left(v_{q-p}, \epsilon_{0}\right)<t\right\}=\left\{q \in V: v_{q-p}(t)>1-t\right\} .
$$


The strong neighbourhood system for $V$ is the union $\bigcup_{p \in V} \mathcal{N}_{p}(\lambda)$ where $\mathcal{N}_{p}=$ $\left\{N_{p}(\lambda): \lambda>0\right\}$. The strong neighborhood system for $V$ determines a Hausdorff topology for $V$.

A linear map $T:\left(V, \nu, \tau, \tau^{*}\right) \rightarrow\left(V^{\prime}, v^{\prime}, \sigma, \sigma^{*}\right)$, is said to be strongly bounded, if there exists a constant $k>0$ such that, for all $p \in V$ and $x>0$,

$$
\nu_{T p}^{\prime}(x) \geq \nu_{p}(x / k) .
$$

\section{DEFINITION 2.7}

A Menger PN space is a PN space $\left(V, v, \tau, \tau^{*}\right)$ in which $\tau=\tau_{T}$ and $\tau^{*}=\tau_{T^{*}}$ for some $t$-norm $T$ and its $t$-conorm $T^{*}$. It will be denoted by $(V, v, T)$.

\section{DEFINITION 2.8}

Let $\left(V, \nu, \tau, \tau^{*}\right)$ be a PN space. A sequence $\left(p_{n}\right)_{n}$ in $V$ is said to be strongly convergent to $p$ in $V$ if for each $\lambda>0$, there exists a positive integer $N$ such that $p_{n} \in N_{p}(\lambda)$, for $n \geq N$. Also the sequence $\left(p_{n}\right)_{n}$ in $V$ is called a strong Cauchy sequence if, for every $\lambda>0$, there is a positive integer $N$ such that $v_{p_{n}-p_{m}}(\lambda)>1-\lambda$, whenever $m, n>N$. A PN space $\left(V, v, \tau, \tau^{*}\right)$ is said to be strongly complete in the strong topology if and only if every strong Cauchy sequence in $V$ is strongly convergent to a point in $V$.

Lemma 2.9 [2]. If $|\alpha| \leq|\beta|$, then $v_{\beta p} \leq v_{\alpha p}$ for every $p$ in $V$.

\section{DEFINITION 2.10}

Let $\left(V_{1}, v_{1}, \tau, \tau^{*}\right)$ and $\left(V_{2}, \nu_{2}, \tau, \tau^{*}\right)$ be two PN spaces under the same triangle functions $\tau$ and $\tau^{*}$. Let $\sigma$ be a triangle function. The $\sigma$-product of the two PN spaces is the quadruple

$$
\left(V_{1} \times V_{2}, \nu_{1} \sigma v_{2}, \tau, \tau^{*}\right)
$$

where

$$
v_{1} \sigma v_{2}: V_{1} \times V_{2} \rightarrow \Delta^{+}
$$

is a probabilistic semi-norm given by

$$
\left(\nu_{1} \sigma \nu_{2}\right)(p, q):=\sigma\left(\nu_{1}(p), \nu_{2}(q)\right)
$$

for all $(p, q) \in V_{1} \times V_{2}$.

\section{Quotient PN space}

According to [8] (see Definition 12.9.3 in p. 215), one has the following:

\section{DEFINITION 3.1}

A triangle function $\tau$ is sup-continuous if, for every family $\left\{F_{\lambda}: \lambda \in \Lambda\right\}$ of d.f.'s in $\Delta^{-}$ and every $G \in \Delta^{+}$,

$$
\sup _{\lambda \in \Lambda} \tau\left(F_{\lambda}, G\right)=\tau\left(\sup _{\lambda \in \Lambda} F_{\lambda}, G\right) .
$$


In view of Lemma 4.3 .5 of [8], this supremum is in $\Delta^{+}$. An example of a sup-continuous triangle function is $\tau_{T}$, where $T$ is a left continuous $t$-norm.

\section{DEFINITION 3.2}

Let $W$ be a linear subspace of $V$ and denote by $\sim_{W}$ a relation on the set $V$ defined via

$$
p \sim_{W} q \Leftrightarrow p-q \in W
$$

for every $p, q \in V$.

Obviously this relationship is an equivalence relation and therefore the set $V$ is partitioned into equivalence classes, $V / \sim_{W}$.

\section{PROPOSITION 3.3}

Let $\left(V, v, \tau, \tau^{*}\right)$ be a PN space. Suppose that $\tau$ and $\tau^{*}$ are sup-continuous. Let $W$ be a subspace of $V$ and $V / \sim_{W}$ its quotient defined by means of the equivalence relation $\sim_{W}$. Let $v^{\prime}$ be the restriction of $v$ to $W$ and define the mapping $\bar{v}: V / \sim_{W} \rightarrow \Delta^{+}$, for all $p \in V$, by

$$
\bar{v}_{p+W}(x):=\sup _{q \in W}\left\{v_{p+q}(x)\right\}
$$

Then, $\left(W, v^{\prime}, \tau, \tau^{*}\right)$ is a PN space and $\left(V / \sim_{W}, \bar{v}, \tau, \tau^{*}\right)$ is a PPN space.

Proof. The first statement is immediate. The remainder of the theorem is guaranteed by the fact that $W$ is not necessarily closed in the strong topology.

Notice that by Lemma 4.3.5 of [8], $\bar{v}_{p+W}$ is in $\Delta^{+}$.

Hereafter we denote by $p_{W}$ the subset $p+W$ of $V$, i.e. an element of quotient, and the strong neighbourhood of $p_{W}$ by $N_{p_{W}}^{\prime}(t)$.

Theorem 3.4. Let $W$ be a linear subspace of $V$. Then the following statements are equivalent:

(a) $\left(V / \sim_{W}, \bar{v}, \tau, \tau^{*}\right)$ is a PN space;

(b) $W$ is closed in the strong topology of $\left(V, \nu, \tau, \tau^{*}\right)$.

Proof. Let $\left(V, \nu, \tau, \tau^{*}\right)$ be a PN space. For every $p$ in the closure of $W$ and for each $n \in \mathbb{N}$ choose $q_{n} \in N_{p}(1 / n) \cap W$. Then

$$
\bar{v}_{p_{W}}(1 / n)=\sup _{q \in W} v_{p+q}(1 / n) \geq v_{p-q_{n}}(1 / n)>1-1 / n,
$$

and hence, $d_{S}\left(\bar{v}_{p_{W}}, \varepsilon_{0}\right)<1 / n$. Thus $p_{W}=W$ and hence, $p \in W$ and $W$ is closed.

Conversely, if $W$ is closed, let $p \in V$ be such that $\bar{v}_{p_{W}}=\varepsilon_{0}$. If $p \notin W$, then $N_{p}(t) \cap$ $W=\varnothing$, for some $t>0$. That is to say, for every $q \in W, v_{p-q}(t) \leq 1-t$. Therefore $\bar{v}_{p_{W}}(t)=\sup _{q \in W} v_{p+q}(t) \leq 1-t$, which is a contradiction.

It is of interest to know whether a PN space can be obtained from a PPN space. An affirmative answer is provided by the following proposition. 


\section{PROPOSITION 3.5}

Let $\left(V, \nu, \tau, \tau^{*}\right)$ be a PPN space and define

$$
C=\left\{p \in V: v_{p}=\varepsilon_{0}\right\} .
$$

Then $C$ is the smallest closed subspace of $\left(V, \nu, \tau, \tau^{*}\right)$.

Proof. If $p, q \in C$, then $p+q \in C$ because $v_{p+q} \geq \tau\left(v_{p}, v_{q}\right)=\varepsilon_{0}$. Now suppose $p \in C$. For $\alpha \in[0,1]$ one has $v_{\alpha p} \geq v_{p}$ by Lemma 2.9. For $\alpha>1$, let $k=[\alpha]+1$. Then, using the iterates of (N3) one has, $v_{k p} \geq \tau^{k-1}\left(v_{p}, \ldots, v_{p}\right)=\varepsilon_{0}$. By the above-mentioned lemma one has $v_{\alpha p} \geq v_{k p}$. As a consequence, $\alpha p$ belongs to $C$ for all $\alpha \in \mathbf{R}$.

Furthermore it is easy to check that the set $C$ is closed because of the continuity of the probabilistic norm, $v$ (see Theorem 1 in [2]).

Now, let $W$ be a closed linear subspace of $V$ and $p \in C$. Suppose that for some $t>0$, $N_{p}(t) \cap W=\varnothing$, then $v_{p}(t) \leq 1-t$, which is a contradiction; hence $C \subseteq W$.

Remark 3.6. Moreover, with $V$ and $C$ as in Proposition 3.5, for all $p \in V$ and $r \in C$, one has

$$
\bar{v}_{p_{W}} \geq v_{p}=v_{p+r-r} \geq \tau\left(v_{p+r}, v_{-r}\right)=v_{p+r} .
$$

Thus the probabilistic norm $\bar{\nu}$ in $\left(V / \sim_{C}, \bar{v}, \tau, \tau^{*}\right)$ coincides with that of $\left(V, \nu, \tau, \tau^{*}\right)$.

Example 3.7. Let $(V, v, T)$ be a Menger PN space. Suppose that $W$ is a closed subspace of $V$, and $V / \sim_{W}$ its quotient. Then $\left(W, v^{\prime}, T\right)$ and $\left(V / \sim_{W}, \bar{v}, T\right)$ are Menger PN spaces.

\section{COROLLARY 3.8}

Let $\left(V, \nu, \tau, \tau^{*}\right)$ be a Šerstnev PN space. Suppose that $\tau$ is sup-continuous. Let $W$ be a closed subspace of $V$ and $V / \sim_{W}$ its quotient. Then, $\left(W, v^{\prime}, \tau, \tau^{*}\right)$ and $\left(V / \sim_{W}, \bar{v}, \tau, \tau^{*}\right)$ are Šrstnev PN spaces.

Theorem 3.9. Let $\left(V, \nu, \tau, \tau^{*}\right)$ be a PN space. Suppose that $\tau$ and $\tau^{*}$ are sup-continuous. Let $W$ be a closed subspace of $V$ with respect to the strong topology of $\left(V, \nu, \tau, \tau^{*}\right)$. Let

$$
\pi: V \rightarrow V / \sim_{W}
$$

be the canonical projection. Then $\pi$ is strongly bounded, open, and continuous with respect to the strong topologies of $\left(V, \nu, \tau, \tau^{*}\right)$ and $\left(V / \sim_{W}, \bar{\nu}, \tau, \tau^{*}\right)$. In addition, the strong topology and the quotient topology on $V / \sim_{W}$, induced by $\pi$, coincide.

Proof. One has that $\bar{v}_{p_{W}} \geq v_{p}$ which implies $\pi$ is strongly bounded, and hence continuous (see Theorem 3.3 in [5]).

The map $\pi$ is open because of the equality $\pi\left(N_{p}(t)\right)=N_{p_{W}}^{\prime}(t)$.

Example 3.10. Let $(V,\|\cdot\|)$ be a normed space and define $v: V \rightarrow \Delta^{+}$via $v_{p}:=\varepsilon_{\|p\|}$ for every $p \in V$. Let $\tau, \tau^{*}$ be continuous triangle functions such that $\tau \leq \tau^{*}$ and $\tau\left(\varepsilon_{a}, \varepsilon_{b}\right)=$ $\varepsilon_{a+b}$, for all $a, b>0$. For instance, it suffices to take $\tau=\tau_{T}$ and $\tau^{*}=\tau_{T^{*}}$, where $T$ is a continuous $t$-norm and $T^{*}$ is its $t$-conorm. Then $\left(V, v, \tau, \tau^{*}\right)$ is a PN space (see Example 1.1 of [5]). 
Assume that $\tau$ is sup-continuous. Let $\mathrm{W}$ be a closed linear subspace of $V$ with respect to the strong topology of $\left(V, \nu, \tau, \tau^{*}\right)$. By Theorem 3.4, $\left(V / \sim_{W}, \mu, \tau, \tau^{*}\right)$ is a PN space in which, $\mu_{p_{W}}=\sup _{w \in W} \varepsilon_{\|p+w\|}$. On the other hand, if one considers the normed space $\left(V / \sim_{W},\|\cdot\|^{\prime}\right)$, where $\left\|p_{W}\right\|^{\prime}=\inf _{w \in W}\|p+w\|$, then one can easily prove that the PN structure given to the normed space $\left(V / \sim_{W},\|\cdot\|^{\prime}\right)$ by means of $\eta_{p_{W}}:=\varepsilon_{\left\|p_{W}\right\|^{\prime}}$ coincides with $\left(V / \sim_{W}, \eta, \tau, \tau^{*}\right)$.

\section{Completeness results}

Here we study the completeness of a quotient PN space. When a PN space $\left(V, \nu, \tau, \tau^{*}\right)$ is strongly complete, then we say that it is a probabilistic normed Banach (henceforth PNB) space.

Lemma 4.1. Given the $P N$ space $\left(V / \sim_{W}, \bar{\nu}, \tau, \tau^{*}\right)$ in which $\tau$ and $\tau^{*}$ are sup-continuous, let $W$ be a closed subspace of $V$.

(i) If $p \in V$, then for every $\epsilon>0$ there is a $p^{\prime}$ in $V$ such that $p^{\prime}+W=p+W$ and

$$
d_{S}\left(\nu_{p^{\prime}}, \varepsilon_{0}\right)<d_{S}\left(\bar{v}_{p+W}, \varepsilon_{0}\right)+\epsilon .
$$

(ii) If $p$ is in $V$ and $\bar{v}_{p+W} \geq G$ for some d.f. $G \neq \varepsilon_{0}$, then there exists $p^{\prime} \in V$ such that $p+W=p^{\prime}+W$ and $v_{p^{\prime}} \geq \tau\left(\bar{v}_{p+W}, G\right)$.

\section{Proof.}

(i) We know

$$
\bar{v}_{p+W}=\sup \left\{v_{p-q}: q \in W\right\} .
$$

Now, let $q$ be an element of $W$ such that

$$
\bar{v}_{p+W}<v_{p-q}+\frac{\epsilon}{2} .
$$

We put $p-q=p^{\prime}$. Now,

$$
\begin{aligned}
d_{S}\left(\bar{v}_{p+W}, \varepsilon_{0}\right) & =\inf \left\{h>0: \bar{v}_{p+W}\left(h^{+}\right)>1-h\right\} \\
& \geq \inf \left\{h>0: v_{p^{\prime}}\left(h^{+}\right)+\frac{\epsilon}{2}>1-h\right\} \\
& =\inf \left\{h>0: v_{p^{\prime}}\left(h^{+}\right)>1-\left(h+\frac{\epsilon}{2}\right)\right\} \\
& \geq \inf \left\{h>0: v_{p^{\prime}}\left(\left(h+\frac{\epsilon}{2}\right)^{+}\right)>1-\left(h+\frac{\epsilon}{2}\right)\right\} \\
& >d_{S}\left(v_{p^{\prime}}, \varepsilon_{0}\right)-\epsilon .
\end{aligned}
$$

(ii) Because of the definition of supremum and sup-continuity of $\tau$, there exists a $q_{n} \in W$ such that $q_{n} \rightarrow q$ if $n \rightarrow+\infty$ and

$$
v_{p+q_{n}}>\tau\left(\bar{v}_{p_{W}}, \varepsilon_{0}\right)-\frac{1}{n} \geq \tau\left(\bar{v}_{p_{W}}, G\right)-\frac{1}{n} .
$$

Now it is enough to put $p^{\prime}=p+q$ and see that, when $n \rightarrow+\infty$, one has $v_{p+q} \geq$ $\tau\left(\bar{v}_{p_{W}}, G\right)$. 
Let $p, q$ be elements of $V$ such that $d_{S}\left(v_{(p-q)+W}, \varepsilon_{0}\right)<\delta$ for some positive $\delta$. By Lemma 4.1, there is a $q^{\prime} \in V$ such that $\left(p-q^{\prime}\right)+W=(p-q)+W$ and

$$
d_{S}\left(\nu_{p-q^{\prime}}, \varepsilon_{0}\right)<\delta .
$$

Theorem 4.2. Let $W$ be a closed subspace of $V$ and suppose that $\left(V, v, \tau, \tau^{*}\right)$ is a PNB space with $\tau$ and $\tau^{*}$ sup-continuous. Then, $\left(V / \sim_{W}, \bar{v}, \tau, \tau^{*}\right)$ is also a PNB space.

Proof. Let $\left(a_{n}\right)$ be a strong Cauchy sequence in $\left(V / \sim_{W}, \bar{v}, \tau, \tau^{*}\right)$, i.e. for every $\delta>0$, there exists $n_{0}=n_{0}(\delta) \in \mathbf{N}$ such that, for all $m, n>n_{0}$,

$$
d_{S}\left(\bar{v}_{a_{n}-a_{m}}, \varepsilon_{0}\right)<\delta .
$$

Now, define a strictly decreasing sequence $\left(\delta_{n}\right)$ with $\delta_{n}>0$ in the following way: let $\delta_{1}>0$ be such that $\tau\left(B_{d_{S}}\left(\varepsilon_{0} ; \delta_{1}\right) \times B_{d_{S}}\left(\varepsilon_{0} ; \delta_{1}\right)\right) \subseteq B_{d_{S}}\left(\varepsilon_{0} ; 1\right)$ where $B_{d_{S}}\left(\varepsilon_{0} ; \lambda\right)=\{F \in$ $\left.\Delta^{+} ; d_{S}\left(F, \varepsilon_{0}\right)<\lambda\right\}$. For $n>1$, define $\delta_{n}$ by induction in such a manner that

$$
\tau\left(B_{d_{S}}\left(\varepsilon_{0} ; \delta_{n}\right) \times B_{d_{S}}\left(\varepsilon_{0} ; \delta_{n}\right)\right) \subseteq B_{d_{S}}\left(\varepsilon_{0} ; \min \left(\frac{1}{n}, \delta_{n-1}\right)\right)
$$

There is a subsequence $\left(a_{n_{i}}\right)$ of $\left(a_{n}\right)$ with

$$
d_{S}\left(\bar{v}_{a_{n_{i}}-a_{n_{i+1}}}, \varepsilon_{0}\right)<\delta_{i+1}
$$

Because of the definition of the canonical projection $\pi$ one can say that $\pi^{-1}\left(N_{p_{W}}^{\prime}(t)\right)=$ $N_{p}(t)$ and consequently $\pi^{-1}\left(a_{n_{i}}\right)=x_{i}$ exists. Inductively, from Lemma 4.1 we can find $x_{i} \in V$ such that $\pi\left(x_{i}\right)=a_{n_{i}}$ and then

$$
d_{S}\left(v_{x_{i}-x_{i+1}}, \varepsilon_{0}\right)<\delta_{i+1}
$$

holds. We claim that $\left(x_{i}\right)$ is a strong Cauchy sequence in $\left(V, \nu, \tau, \tau^{*}\right)$. By applying the relations (1), (2) and (3) to $i=m-1$ and $i=m-2$, and using Lemma 4.3.4 of [8], one obtains the inequalities

$$
\begin{aligned}
d_{S}\left(v_{x_{m}-x_{m-2}}, \varepsilon_{0}\right) & \leq d_{S}\left(\tau\left(v_{x_{m-1}-x_{m}}, v_{x_{m-2}-x_{m-1}}\right), \varepsilon_{0}\right) \\
& <\min \left(\frac{1}{m-1}, \delta_{m-2}\right) .
\end{aligned}
$$

Following this reasoning, we obtain that $d_{S}\left(v_{x_{m}-x_{n}}, \varepsilon_{0}\right)<1 / n$ and therefore, $\left(x_{i}\right)$ is a strong Cauchy sequence. Since it was assumed that $\left(V, \nu, \tau, \tau^{*}\right)$ is strongly complete, $\left(x_{i}\right)$ is strongly convergent and hence, by the continuity of $\pi,\left(a_{n_{i}}\right)$ is also strongly convergent. From this and taking into account the continuity of $\tau$ and Lemma 4.3.4 of [8], one sees that the whole sequence $\left(a_{n}\right)$ strongly converges.

The converse of the above theorem also holds.

Theorem 4.3. Let $\left(V, \nu, \tau, \tau^{*}\right)$ be a PN space in which $\tau$ and $\tau^{*}$ sup-continuous, and let $\left(V / \sim_{W}, \bar{v}, \tau, \tau^{*}\right)$ be its quotient space with respect to the closed subspace $W$. If any two of the three spaces $V, W$ and $V / \sim_{W}$ are strongly complete, so is the third. 
Proof. If $V$ is a strongly complete PN space, so are $V / \sim_{W}$ and $W$. Therefore all one needs to check is that $V$ is strongly complete whenever both $W$ and $V / \sim_{W}$ are strongly complete. Suppose $W$ and $V / \sim_{W}$ are strongly complete PN spaces and $\left(p_{n}\right)$ be a strong Cauchy sequence in $V$. Since

$$
\bar{v}_{\left(p_{m}-p_{n}\right)+W} \geq v_{p_{m}-p_{n}}
$$

whenever $m, n \in \mathbf{N}$, the sequence $\left(p_{n}+W\right)$ is strong Cauchy in $V / \sim_{W}$ and, therefore, it strongly converges to $q+W$ for some $q \in V$. Thus there exists a sequence of d.f's $\left(H_{n}\right)$ such that $H_{n} \longrightarrow \varepsilon_{0}$ and $\bar{\nu}_{\left(p_{n}-q\right)+W}>H_{n}$. Now by Lemma 4.1 there exists $\left(q_{n}\right)$ in $V$ such that $q_{n}+W=\left(p_{n}-q\right)+W$ and

$$
v_{q_{n}}>\tau\left(\bar{v}_{\left(p_{n}-q\right)+W}, H_{n}\right) \text {. }
$$

Thus $v_{q_{n}} \longrightarrow \varepsilon_{0}$ and consequently $q_{n} \longrightarrow \theta$. Therefore $\left(p_{n}-q_{n}-q\right)$ is a strong Cauchy sequence in $W$ and is strongly convergent to a point $r \in W$ and implies that $\left(p_{n}\right)$ strongly converges to $r+q$ in $V$. Hence $V$ is strongly complete.

Theorem 4.4. Let $\left(V_{1}, v^{1}, \tau, \tau^{*}\right), \ldots,\left(V_{n}, v^{n}, \tau, \tau^{*}\right)$ be PNB spaces in which $\tau$ and $\tau^{*}$ are sup-continuous. Suppose that there is a triangle function $\sigma$ such that $\tau^{*} \gg \sigma$ and $\sigma \gg \tau$. Then their $\sigma$-product is a PNB space.

Proof. One proves for $n=2$ (see Theorem 2 in [3]), and then we apply induction for an arbitrary $n$. Since the quotient norm of

$$
\frac{V_{1} \times V_{2}}{V_{1} \times \theta_{2}}\left(\simeq V_{2}\right)
$$

is the same as $v^{2}$ and the restriction of the product norm of $V_{1} \times V_{2}$ to $V_{1} \times \theta_{2}\left(\simeq V_{1}\right)$ is the same as $v^{1}$ (see [3]), and in view of Theorem 4.3, the proof is complete.

By Theorem 3.9 the following corollaries can be proved easily.

\section{COROLLARY 4.5}

Under the assumptions of Proposition 3.3 and if $W$ is a closed subset of $V$, the probabilistic norm $\bar{v}: V / \sim_{W} \rightarrow \Delta^{+}$in $\left(V / \sim_{W}, \bar{v}, \tau, \tau^{*}\right)$ is uniformly continuous.

Proof. Let $\eta$ be a positive real number, $\eta>0$. By Theorem 3.9 there exists a pair $\left(p^{\prime}, q^{\prime}\right)$ in $(V \times V)$ such that $d_{S}\left(\bar{v}_{\pi\left(p-p^{\prime}\right)}, \varepsilon_{0}\right)<\eta$ and $d_{S}\left(\bar{v}_{\pi\left(q-q^{\prime}\right)}, \varepsilon_{0}\right)<\eta$, whenever $d_{S}\left(v_{p-p^{\prime}}, \varepsilon_{0}\right)<\eta$ and $d_{S}\left(v_{q-q^{\prime}}, \varepsilon_{0}\right)<\eta$.

On the other hand, we have

$$
\bar{v}_{\pi\left(p^{\prime}-q^{\prime}\right)} \geq \tau\left(\tau\left(\bar{v}_{\pi\left(p-p^{\prime}\right)}, \bar{\nu}_{\pi\left(q-q^{\prime}\right)}\right), \bar{\nu}_{\pi(p-q)}\right)
$$

and

$$
\bar{v}_{\pi(p-q)} \geq \tau\left(\tau\left(\bar{\nu}_{\pi\left(p-p^{\prime}\right)}, \bar{v}_{\pi\left(q-q^{\prime}\right)}\right), \bar{\nu}_{\pi\left(p^{\prime}-q^{\prime}\right)}\right) .
$$

Thus, from the relationship (12.1.5) and Lemma 12.2.1 in [8] it follows that for any $h>0$ there is an appropriate $t>0$ such that

$$
d_{S}\left(\bar{v}_{\pi(p-q)}, \bar{v}_{\pi\left(p^{\prime}-q^{\prime}\right)}\right)<h,
$$


whenever $p^{\prime} \in N_{p}(\eta)$ and $q^{\prime} \in N_{q}(\eta)$. This implies that $\bar{v}$ is a uniformly continuous mapping from $V / \sim_{W}$ into $\Delta^{+}$.

Also the inequality $d_{S}\left(\bar{\nu}_{\pi\left((p+q)-\left(p^{\prime}+q^{\prime}\right)\right)}, \varepsilon_{0}\right) \leq d_{S}\left(\nu_{(p+q)-\left(p^{\prime}+q^{\prime}\right)}, \varepsilon_{0}\right)$ implies that $\left(V / \sim_{W},+\right)$ is a topological group.

\section{COROLLARY 4.6}

Let $\left(V, \nu, \tau, \tau^{*}\right)$ be a PN space such that $\tau^{*}$ is Archimedean, $\tau$ and $\tau^{*}$ are sup-continuous, and $\nu_{p} \neq \varepsilon_{\infty}$ for all $p \in V$. If we define quotient probabilistic norm via Proposition 3.3, then $\left(V / \sim_{W}, \bar{v}, \tau, \tau^{*}\right)$ is a PPN space where the scalar multiplication is a continuous mapping from $R \times V / \sim_{W}$ into $V / \sim_{W}$.

Proof. For any $p \in V$ and $\alpha, \beta \in R$ we know $d_{S}\left(\bar{v}_{\pi(\alpha p)}, \nu_{\pi(\beta p)}\right)$ is small whenever $d_{S}\left(\bar{v}_{\pi((\alpha-\beta) p)}, \varepsilon_{0}\right)$ is small. But

$$
d_{S}\left(\bar{\nu}_{\pi((\alpha-\beta) p)}, \varepsilon_{0}\right) \leq d_{S}\left(\nu_{(\alpha-\beta) p}, \varepsilon_{0}\right)
$$

and by Lemma 3 of [2], $d_{S}\left(\nu_{(\alpha-\beta) p}, \varepsilon_{0}\right)$ is small whenever $|\alpha-\beta|$ is small.

\section{Acknowledgments}

The authors would like to thank the referee for giving useful comments and suggestions for the improvement of this paper. The authors wish to thank C Sempi for his helpful suggestions. The first author was supported by grants from the Ministerio de Ciencia y Tecnología (BFM2005-06522) and from the Junta de Andalucía (CEC-JA FQM-197), Spain.

\section{References}

[1] Alsina C, Schweizer B and Sklar A, On the definition of a probabilistic normed space, Aequationes Math. 46 (1993) 91-98

[2] Alsina C, Schweizer B and Sklar A, Continuity properties of probabilistic norms, J. Math. Anal. Appl. 208 (1997) 446-452

[3] Lafuerza-Guillén B, Finite products of probabilistic normed spaces, Rad. Mat. 13 (2004) 111-117

[4] Lafuerza-Guillén B, Rodríguez-Lallena J A and Sempi C, Completion of probabilistic normed spaces, Int. J. Math. Math. Sci. 18 (1995) 649-652

[5] Lafuerza-Guillén B, Rodríguez-Lallena J A and Sempi C, A study of boundedness in probabilistic normed spaces, J. Math. Anal. Appl. 232 (1999) 183-196

[6] Lafuerza-Guillén B, Rodríguez-Lallena J A and Sempi C, Probabilistic norms for linear operators, J. Math. Anal. Appl. 220 (1998) 462-476

[7] Saadati R and Amini M, D-boundedness and $D$-compactness in finite dimensional probabilistic normed spaces, Proc. Indian Acad. Sci. (Math. Sci.) 115 (2005) 483-492

[8] Schweizer B and Sklar A, Probabilistic metric spaces (New York: North-Holland) (1983)

[9] Sibley D A, A metric for weak convergence of distribution functions, Rocky Mountain J. Math. 1 (1971) 427-430 